\title{
ALGEBRAS SATISFYING CONGRUENCE RELATIONS
}

\author{
RAYMOND COUGHLIN, MICHAEL RICH AND ARMIN THEDY
}

ABSTRACT. It is shown that the classical nonassociative algebras which have an identity element can be defined in terms of congruence relations modulo the base field.

1. Introduction. In an earlier note [2] two of the authors have shown that if an algebra $A$ with identity element 1 over a field $F$ satisfies the property that $(x y) z-x(y z) \in F \cdot 1$ for all $x, y, z$ in $A$, then $A$ is an associative algebra. Here we consider the similar question for the classical nonassociative algebras. Recall than an alternative algebra is one which satisfies $x^{2} y-x(x y)=y x^{2}-(y x) x=0$ for all elements $x, y$ and a (linear) Jordan algebra is a commutative algebra over a field of characteristic $\neq 2$ which satisfies $(x y) x^{2}-x\left(y x^{2}\right)=0$ for all elements $x, y$. In our main results we show that if $A$ is an algebra with identity element 1 over a field $F$ such that $x y-y x \in F \cdot 1$ and $(x y) x^{2}-x\left(y x^{2}\right) \in F \cdot 1$ for all elements $x$ and $y$, then $A$ is a Jordan algebra. Also if the characteristic of $F$ is not 3 and if $x(x y)-x^{2} y \in F \cdot 1$ and $(y x) x-y x^{2} \in F \cdot 1$ for all elements $x$ and $y$, then $A$ is an altemative algebra. Similar results for strongly alternative algebras, noncommutative Jordan algebras, and power-associative algebras are established.

As usual $(x, y, z)$ denotes $(x y) z-x(y z)$ and $[x, y]$ denotes $x y-y x$. Wherever convenient we will write $a \equiv 0 \bmod F$ instead of $a \in F \cdot 1$. Also, throughout we shall use the term "algebra" to mean a not necessarily associative algebra with identity element 1 over a field $F$.

Our results depend on the Teichmüller or 5-identity:

$$
x(y, z, w)+(x, y, z) w=(x y, z, w)-(x, y z, w)+(x, y, z w)
$$

which holds in any nonassociative ring. We also rely heavily on the ability to linearize identities [3], [5] and on the linear independence of various elements of our algebra. Thus, we restrict our attention to algebras over fields.

Received by the editors June 14, 1974.

AMS (MOS) subject classifications (1970). Primary 17A05, 17A20, 17A30, 17 D05.

Key words and phrases. Alternative algebra, strongly alternative algebra, Jordan algebra, noncommutative Jordan algebra, flexible, Jordan-admissible. 
2. The alternative case. Recall that an algebra is called flexible if $(x, y, x)=0$ for all elements $x, y$.

Lemma 1. If $A$ is an algebra in which $\left(x, x^{2}, x\right) \equiv 0 \bmod F$ for all $x$, $y$ in $A$, then $2(x, x, x)=0$ for all $x$ in $A$.

Proof. The result is trivially true if $x \in F \cdot 1$. Assume now that $x \notin \xi$ $F \cdot 1$. In $\left(x, x^{2}, x\right) \equiv 0 \bmod F$, replace $x$ by $x+1$ to yield $2(x, x, x) \equiv 0$ $\bmod F$. Next, linearize the relation $2(x, x, x) \equiv 0 \bmod F$ to get $2\left(x^{2}, x, x\right)$ $+2\left(x, x^{2}, x\right)+2\left(x, x, x^{2}\right) \equiv 0 \bmod F$. Thus $2\left(x^{2}, x, x\right)+2\left(x, x, x^{2}\right) \equiv 0 \bmod$ $F$. By the 5-identity

$$
x(x, x, x)+(x, x, x) x=\left(x^{2}, x, x\right)-\left(x, x^{2}, x\right)+\left(x, x, x^{2}\right) .
$$

Since $2(x, x, x) \in F \cdot 1$ we have $4 x(x, x, x) \in F \cdot 1$. But $x \notin F \cdot 1$. Therefore $4(x, x, x)=0$ and so $2(x, x, x)=0$.

Lemma 2. If $A$ is an algebra in which $(x, x, y) \equiv(y, x, x) \equiv 0 \bmod F$ for all $x, y$ in $A$, then $A$ is flexible.

Proof. Expand $(x+y, x+y, x) \in F \cdot 1$ to get $(x, y, x) \in F \cdot 1$. Thus Lemma 1 applies.

By the 5-identity we have

$$
\left(x^{2}, x, y\right)-\left(x, x^{2}, y\right)+(x, x, x y)-(x, x, x) y=x(x, x, y)
$$

and

$$
\left(y, x, x^{2}\right)-\left(y, x^{2}, x\right)+(y x, x, x)-y(x, x, x)=(y, x, x) x .
$$

Now add equations (1) and (2). Since $(x, y, x) \in F \cdot 1$ and $2(x, x, x)=$ 0 it follows that the left side of the resulting equation is in $F \cdot 1$. Therefore $x(x, x, y)+(y, x, x) x \in F \cdot 1 \cap F \cdot x$. Thus we have

$$
(x, x, y)+(y, x, x)=0 .
$$

From the 5-identity again, we get

$$
\left(x^{2}, y, x\right)=x(x, y, x)+(x, x, y) x+(x, x y, x)-(x, x, y x) .
$$

Therefore $\left(x^{2}, y, x\right) \in F \cdot 1+F \cdot x$. This gives $\left(x^{2}, x, y\right) \in F \cdot 1+$ $F \cdot x$ and $\left(x, x^{2}, y\right) \in F \cdot 1+F \cdot x$. From (1) we can now conclude that $(x, x, x) y \in F \cdot 1+F \cdot x$ for all $x, y$ in $A$. Thus $(x, x, x)=0$ for all $x$ in $A$. For if $\operatorname{dim} A>2$, an element $y$ can be chosen such that $y \notin F \cdot 1+$ $F \cdot x$ whereas if $\operatorname{dim} A \leq 2$, it is automatic that $A$ is associative. 
Now if $F$ contains more than two elements, we can linearize $(x, x, x)$ $=0$ to obtain $(x, x, y)+(y, x, x)+(x, y, x)=0$ and from (3) it follows that $A$ is flexible. If $F$ contains only two elements, we imbed $F$ in a larger field $\bar{F}$ and consider the scalar extension $\bar{A}=A \otimes_{F} \bar{F}$. Since $(x, x, y) \epsilon$ $F \cdot 1$ and $(y, x, x) \in F \cdot 1$ for all $x, y$ in $F$, it is immediate that $(\bar{x}, \bar{x}, \bar{y})$ $\epsilon \bar{F} \cdot 1$ for all $\bar{x}, \bar{y}$ in $\bar{A}$. Consequently $\bar{A}$ is flexible and thus $A$ is flexible also.

San Soucie [6] has defined a strongly right alternative algebra to be a right alternative algebra $((y, x, x)=0)$ satisfying the identity $((x y) z) y=$ $x((y z) y)$. Thedy [0] has shown that for a right alternative algebra this is equivalent to $\left(y, x, x^{2}\right)=0$ in all extensions. We now prove

Lemma 3. If. $A$ is an algebra in which $(y, x, x) \equiv\left(y, x, x^{2}\right) \equiv 0 \bmod F$ for all $x, y$ in $A$, then $(y, x, x)=\left(y, x, x^{2}\right)=0$ for all $x, y$ in $A$.

Proof. From the 5-identity we have $y(x, x, x)+(y, x, x) x=(y x, x, x)-$ $\left(y, x^{2}, x\right)+\left(y, x, x^{2}\right)$ and the right side is in $F \cdot 1$ by hypothesis. Therefore we have

$$
y(x, x, x)+(y, x, x) x \equiv 0 \bmod F \cdot 1 \text { for all } x, y \text { in } A .
$$

Now we may assume that there exists a $y$ in $A$ which is linearly independent of 1 and $x$ for otherwise $A$ would be associative. Thus $(x, x, x)$ $=0$ for all $x$ in $A$. Consequently $(y, x, x) x \in F \cdot 1 \cap F \cdot x$ for all $y$ in $A$ from which we conclude that $(y, x, x)=0$.

Irrespective of the number of elements in $F$ we may linearize the identity $\left(y, x^{2}, x\right) \equiv 0 \bmod F$ to get $2\left(y, x^{3}, x\right) \equiv 0 \bmod F$. Therefore the right side of

$$
y\left(x, x^{2}, x\right)+\left(y, x, x^{2}\right) x=\left(y x, x^{2}, x\right)-\left(y, x^{3}, x\right)+\left(y, x, x^{3}\right)
$$

is in $F \cdot 1$ so that we have $y\left(x, x^{2}, x\right)+\left(y, x, x^{2}\right) x \equiv 0 \bmod F$ for all $x, y$ in $A$ and by the same argument as before we arrive at $\left(y, x, x^{2}\right)=0$.

We remark that if $F$ has at least three elements and $(y, x, x) \equiv\left(y, x, x^{2}\right)$ $\equiv 0 \bmod F$, then the algebra is strongly right alternative. For by the lemma, $(y, x, x)=\left(y, x, x^{2}\right)=0$ for all $x, y$ in $A$, and since $F$ has at least three elements, these identities hold in all extensions.

We are now able to prove our first main result.

Theorem 1. If $A$ is an algebra in which $(x, x, y) \equiv(y, x, x) \equiv 0 \bmod F$ for all $x, y$ in $F$ and if the characteristic of $F \neq 3$, then $A$ is an alternative algebra. 
Proof. From the 5-identity and Lemma 2 we have $x(y, x, x) \equiv\left(x, y, x^{2}\right)$ $\bmod F$ and $x(x, x, y) \equiv\left(x^{2}, x, y\right)-\left(x, x^{2}, y\right) \bmod F$. We add these congruences to get

$$
x[(y, x, x)+(x, x, y)] \equiv 3\left(x, y, x^{2}\right) \bmod F
$$

or, again by Lemma $2,3\left(x, y, x^{2}\right) \equiv 0 \bmod F$. Since characteristic $F \neq 3$, we have $\left(x, y, x^{2}\right) \equiv 0 \bmod F$. Then from Lemma 3 it follows that $(y, x, x)$ $=0$. Linearization of the flexible law gives $(x, x, y)=0$. Therefore $A$ is alternative.

3. The Jordan case. Recall that a ring $R$ is called noncommutative Jordan if it is flexible and satisfies the Jordan law $\left(x, y, x^{2}\right)=0$. If $R$ is a ring in which to each $a \in R$ there is a unique $b \in R$ such that $2 b=a$, we can define the attached ring $R^{+}$to be the same additive group as $R$ with multiplication in $R^{+}$given by $x \cdot y=1 / 2(x y+y x)$ where $x y$ denotes the multiplication in $R$. In particular this applies to an algebra over a field of characteristic $\neq 2$.

Theorem 2. If $A$ is an algebra over a field $F$ of characteristic $\neq 2$ in which $[x, y] \equiv\left(x, y, x^{2}\right) \equiv 0 \bmod F$ for all $x, y$ in $A$, then $A$ is a Jordan algebra.

Proof. Since $A$ contains an identity element 1 , linearization of $\left(x, y, x^{2}\right) \equiv 0 \bmod F$ yields $(x, y, x) \equiv 0 \bmod F[8]$. On the other hand $[x y, x]=x[y, x]+(x, y, x)$ holds in any ring. Therefore we get $x[y, x] \epsilon$ $F \cdot \ln F \cdot x$ so that we can conclude that $[x, y]=0$ and $A$ is commutative. Hence $A$ is flexible and

$$
(x, y, z)+(y, z, x)+(z, x, y)=0 \text { for all } x, y, z \text { in } A \text {. }
$$

Thus $\left(x^{2}, y^{2}, x\right)=-\left(y^{2}, x, x^{2}\right)-\left(x, x^{2}, y^{2}\right)$. Linearization of the hypothesis gives $\left(x^{2}, y, z\right)+2(x z, y, x) \equiv 0 \bmod F$ and thus the right side of the last equation is congruent to $2\left(x^{2} y, x, y\right)+2\left(y, x_{-}^{2}, x y\right) \bmod F$. Thus we have

$$
\left(x^{2}, y^{2}, x\right) \equiv 2\left(x^{2} y, x, y\right)+2\left(y, x^{2}, x y\right) \bmod F \text { for all } x, y \text { in } A \text {. }
$$

Now by (6) again we have

$$
2\left(x^{2} y, x, y\right)+2\left(y, x^{2}, x y\right)=2\left(x^{2} y, x, y\right)-2\left(x^{2}, y x, y\right)+2\left(x^{2}, y, x y\right) .
$$

Therefore, we have 
(8) $\left(x^{2}, y^{2}, x\right) \equiv 2\left(x^{2} y, x, y\right)-2\left(x^{2}, y x, y\right)+2\left(x^{2}, y, x y\right) \bmod F$

for all $x, y$ in $A$.

Thus, by the 5-identity and flexibility we arrive at: $\left(x^{2}, y^{2}, x\right) \equiv$ $2\left(x^{2}, y, x\right) y \bmod F$. Hence $2\left(x^{2}, y, x\right) y \in F \cdot 1 \cap F \cdot y$ for all $x, y$ in $A$. It follows that $\left(x^{2}, y, x\right)=0$ and $A$ is a Jordan algebra.

An algebra $R$ is called Jordan admissible if $R^{+}$is a Jordan algebra.

Corollary 1. If $A$ is an algebra over a field $F$ of characteristic $\neq 2$ in which $\left(x, y, x^{2}\right) \equiv 0 \bmod F$ for all $x, y$ in $A$, then $A$ is a Jordan admis. sible algebra.

Proof. Again it follows from the hypothesis that $(x, y, x) \equiv 0 \bmod F$. Therefore Lemma 1 yields $(x, x, x)=0$ and its linearized version $(x, x, y)$ $+(x, y, x)+(y, x, x)=0$. Now it is straightforward that

$$
\begin{aligned}
4\left[(x \cdot y) \cdot x^{2}-x \cdot\left(y \cdot x^{2}\right)\right]= & \left(x, y, x^{2}\right)-\left(x^{2}, y, x\right)+\left(y, x, x^{2}\right) \\
& -\left(x^{2}, x, y\right)+\left(x, x^{2}, y\right)-\left(y, x^{2}, x\right) .
\end{aligned}
$$

Hence

$$
\begin{aligned}
4\left[(x \cdot y) \cdot x^{2}-x \cdot\left(y \cdot x^{2}\right)\right] \equiv 2\left(y, x, x^{2}\right)-2\left(y, x^{2}, x\right) \bmod F & \quad(\operatorname{since}(x, y, x) \in F \cdot 1) \\
& \equiv-2(y x, x, x)+2(y, x, x) x \bmod F \quad(5 \text {-identity) } \\
& \equiv 2(x, x, y x)+2(y, x, x) x \bmod F \\
& \equiv 2 x(x, y, x)+2(x, x, y) x+2(y, x, x) x \bmod F \\
& \equiv 2[(x, y, x)+(x, x, y)+(y, x, x)] x \bmod F \\
& =0
\end{aligned}
$$

since $(x, y, x)+(x, x, y)+(y, x, x)=0$. Therefore $(x \cdot y) \cdot x^{2}-x \cdot\left(y \cdot x^{2}\right)$ $\equiv 0 \bmod F$. Hence $A^{+}$satisfies the conditions of Theorem 2 and is a Jordan algebra. Therefore $A$ is Jordan admissible.

Since a flexible, Jordan admissible algebra is noncommutative Jordan [7], the following corollary is immediate.

Corollary 2. If $A$ is a flexible algebra over a field $F$ of characteristic $\neq 2$ in which $\left(x, y, x^{2}\right) \equiv 0 \bmod F$ for all $x, y$ in $A$, then $A$ is a noncommutative Jordan algebra.

Examples. 1. The following example shows that the result of Corollary 
2 is not true if the algebra is not assumed to be flexible. Let $A$ be the 4-dimensional algebra over a field $F$ of characteristic $\neq 2$ with basis $1, a, b, c$. Define multiplication by $a^{2}=b^{2}=c^{2}=1, a b=-b a=c$, and all other products zero. Then, for all $x, y$ in $A$ one notes that $(x, y, x) \equiv$ $\left(x^{2}, y, x\right) \equiv 0 \bmod F$. However $(a, b, c)+(c, b, a)=2 \neq 0$. Therefore $A$ is not flexible. In addition it is easy to see that $A$ is a simple algebra.

2. There are many examples of simple, power-associative algebras in which $[x, y] \equiv 0 \bmod F$ but which are not commutative. See, for example, Example 2 of [4] and the class of algebras constructed in [1].

3. The following is an example of an algebra $A$ with an idempotent $e$ in the center $C$ of $A$ such that $(x, y, z) \in F \cdot e \subseteq C$ for all $x, y, z$ in $A$, but $A$ is not even power-associative. Let $A$ be the 4-dimensional algebra with basis $e, x, y, z$ and multiplication given by: $x y=y x=z, e^{2}=z x=$ $x z=y z=z y=e$ and all other products zero. Thus, the results of Theorem 1 would be false if the congruences were assumed modulo the center.

4. The power-associative case. In an arbitrary algebra $A$ powers of elements $x$ in $A$ are defined inductively by $x^{n}=x x^{n-1}$. $A$ is called powerassociative if $x^{m} x^{n}=x^{m+n}$ for all $x$ in $A$ and for all positive integers $m, n$. This is easily equivalent to $\left(x^{p}, x^{q}, x^{r}\right)=0$ for all $x, p, q, r$.

Theorem 3. If $A$ is an algebra in which $\left(x^{p}, x^{q}, x^{r}\right) \equiv 0 \bmod F$ for all $x$ in $A$ and all positive integers $p, q, r$, then $A$ is a power-associative algebra.

Proof. Let $x$ be in $A$. Then by the 5-identity we have

$$
x\left(x, x, x^{2}\right)+(x, x, x) x^{2}=\left(x^{2}, x, x^{2}\right)-\left(x, x^{2}, x^{2}\right)+\left(x, x, x^{3}\right) .
$$

By hypothesis the right side is in $F \cdot 1$. Thus $x\left(x, x, x^{2}\right)+(x, x, x) x^{2} \equiv 0$ $\bmod F$. It follows that either $(x, x, x)=0$ or $x^{2} \in F \cdot 1+F \cdot x$. But the latter also implies that $(x, x, x)=0$. Assume inductively that $x^{m} x^{n}=$ $x^{m+n}$ for $3 \leq m+n \leq N$. Now let $m+n=N+1$. If $m=1$, then $x^{m} x^{n}=$ $x^{m+n}$ by definition. If $m>1$, then by the induction hypothesis we have

$$
x^{m} x^{n}=\left(x x^{m-1}\right) x^{n}=\left(x, x^{m-1}, x^{n}\right)+x^{N+1} .
$$

Thus, if $\left(x, x^{m-1}, x^{n}\right)=0$ we are done. By the 5-identity we have $x\left(x^{m-1}, x^{n}, x^{2}\right)+\left(x, x^{m-1}, x^{n}\right) x^{2} \equiv 0 \bmod F$. Now if $\left(x, x^{m-1}, x^{n}\right) \neq 0$, then $x^{2} \in F \cdot 1+F \cdot x$ which implies that $x^{n} \in F \cdot 1+F \cdot x$ and $x^{m-1} \epsilon$ $F \cdot 1+F \cdot x$. Thus, $\left(x, x^{m-1}, x^{n}\right)=0$ (since $\left.(x, x, x)=0\right)$. Therefore $x^{m} x^{n}=x^{N+1}=x^{m+n}$ and $A$ is power-associative. 


\section{REFERENCES}

1. T. Anderson, A note on derivations of commutative algebras, Proc. Amer. Math. Soc. 17 (1966), 1199-1202. MR 34 \# 4309.

2. R. Coughlin and M. Rich, Some associativity conditions for algebras, Amer. Math. Monthly 78 (1971), 1107. MR 45 \# 5176.

3. J. Goldman and S. Kass, Linearization in rings and algebras, Amer. Math. Monthly 76 (1969), 348-355. MR 39 \# 2821.

4. E. Kleinfeld, Quasi-nil rings, Proc. Amer. Math. Soc. 10 (1959), 477-479. MR 21 \# 3469.

5. J. M. Osborn, Varieties of algebras, Advances in Math. 8 (1972), 163-369. MR 44 \#6775.

6. R. L. San Soucie, Right alternative division rings of characteristic two, Proc. Amer. Math. Soc. 6 (1955), 291-296. MR 16, 896; erratum 16, 1331.

7. R. D. Schafer, Noncommutative Jordan algebras of characteristic $\mathbf{0}$, Proc. Amer. Math. Soc. 6 (1955), 472-475. MR 17, 10.

8. - An introduction to nonassociative algebras, Pure and Appl. Math., vol. 22, Academic Press, New York, 1966. MR 35 \# 1643.

9. A. Thedy, Right alternative rings, J. Algebra (to appear).

DEPARTMENT OF MATHEMATICS, TEMPLE UNIYERSITY, PHIL ADELPHIA, PENNSYLVANIA $19 \mathrm{I}_{22}$

MATHEM ATICAL INSTITUTE, UNIVERSITY OF DORTMUND, POSTF ACH 500 (D 46), DO-HOMBRUCH, FEDERAL REPUBLIC OF GERMANY 